\title{
Factors associated with poor pulmonary function: cross-sectional analysis of data from the ERCF
}

\author{
J. Navarro*, M. Rainisio\#, H.K. Harms ${ }^{\Uparrow}$, M.E. Hodson ${ }^{+}$, C. Koch ${ }^{\S}$, G. Mastella ${ }^{f}$, B. Strandvik**, \\ S.G. McKenzie ${ }^{\# \#, ~ o n ~ b e h a l f ~ o f ~ t h e ~ i n v e s t i g a t o r s ~ o f ~ t h e ~ E u r o p e a n ~ E p i d e m i o l o g i c ~ R e g i s t r y ~ o f ~ C y s t i c ~}$ \\ Fibrosis (ERCF)
}

Factors associated with poor pulmonary function: cross-sectional analysis of data from the ERCF. J. Navarro, M. Rainisio, H.K. Harms, M.E. Hodson, C. Koch, G. Mastella, B. Strandvik, S. G. McKenzie, on behalf of the investigators of the European Epidemiologic Registry of Cystic Fibrosis (ERCF). C ERS Journals Ltd 2001.

ABSTRACT: The European Epidemiologic Registry of Cystic Fibrosis began collecting longitudinal data on European cystic fibrosis patients in 1994. A crosssectional analysis was performed to identify the factors associated with low values of $\%$ predicted forced expiratory volume in one second (FEV1) upon patient enrolment.

Data from 7,010 patients aged $\geqslant 6$ yrs were included. Clinical conditions, microbiological isolates and medications reported at enrolment or within the following 180 days were analysed for age-specific associations.

Factors associated with FEV1 that were lower by $>10 \%$ of pred values were: lower weight for age percentiles, haemoptysis, pneumothorax, pulmonary symptoms at presentation, Pseudomonas aeruginosa, Burkholderia cepacia, oral corticosteroids, nonsteroid anti-inflammatory drugs, dornase alfa, oxygen and assisted ventilation and, in patients $>12$ yrs old only, use of airway clearance techniques, inhaled bronchodilators, oral nutritional supplements, pancreatic enzymes and insulin or oral hypoglycaemics. Slightly impaired lung function $(5-10 \%)$ was associated with: diabetes $(\geqslant 18$-yrs-old), gastro-oesophageal reflux, allergic bronchopulmonary aspergillosis, asthma-like symptoms, portal hypertension, Aspergillus spp. and Candida spp. Sex, Haemophilus influenzae and Staphylococcus aureus were not associated with impaired pulmonary status. Regular exercise (especially in older patients) and nasal polyposis were associated with slightly better FEV1.

The results confirm those of previous studies and suggest selective prescribing in sicker patients.

Eur Respir J 2001; 18: 298-305.

\begin{abstract}
*Dept of Gastroenterology and Cystic Fibrosis, Robert Debré Hospital AP/HP, Paris, France, "Btatistics for Research SFR Ltd, Basel, Switzerland, "Munich University Children's Hospital, Munich, Germany, ${ }^{+}$Cystic Fibrosis Dept, Royal Brompton Hospital, London, UK, ${ }^{\S}$ Dept of Paediatrics, Rigshospitalet, University Hospital, Copenhagen, Denmark, Dept of Pulmonary and Digestive Diseases of Developmental Age, Cystic Fibrosis Centre, Verona, Italy, Dept of Paediatrics, Göteborg University, Göteborg, Sweden and ${ }^{\# \text { Pharmaceuti- }}$ cals Division, F. Hoffmann-La Roche Ltd, Basel, Switzerland.
\end{abstract}

Correspondence: J. Navarro, Hôpital Robert Debré, Gastro-entérologie, 48 Boulevard Serrurier, 75019 Paris, France.

Fax: 33140032353

Keywords: Cystic fibrosis

pulmonary function

registry

respiratory function tests

Received: July 282000

Accepted after revision March 102001

The European Epidemiologic Registry of Cystic Fibrosis (ERCF) Advisory Board, an independent team of academics who manage the ERCF with complete editorial freedom, would like to acknowledge financial support from F. Hoffmann-La Roche, Basel, Switzerland for funding the ERCF.
Cystic fibrosis (CF) is the most common hereditary disease in the Caucasian population [1], and results from a mutation in the gene encoding for the $\mathrm{CF}$ transmembrane conductance regulator on chromosome seven [2, 3]. Although it is debilitating, improved understanding and treatment over recent years have led to a dramatic increase in the life expectancy of individuals with this condition [4].

Although the predictive value of pulmonary function for survival in $\mathrm{CF}$ remains controversial [5], all regression models used in the National Canadian Patient Data Registry's study showed that forced expiratory volume in one second (FEV1) was the strongest predictor for survival [6]. Furthermore, FEV1 remains one of the major indicators for lung transplantation [7]. The European Epidemiologic Registry of Cystic Fibrosis (ERCF), sponsored by F. Hoffmann-La Roche Ltd, was established in 1994 to analyse the progression of lung disease with respect to infection rates, other manifestations of $\mathrm{CF}$, and the use of antibiotics and other medications [8, 9]. The ultimate aim was to identify risk factors and interventions that influence the course of pulmonary disease associated with $\mathrm{CF}$.

The aim of this cross-sectional analysis was to identify factors associated with poor pulmonary function (FEV1 \% predicted) in $\mathrm{CF}$ patients at the time of enrolment into the ERCF. The factors examined included patient age, sex, weight for age percentile as a measure for nutritional status, medical 
history, medical status at enrolment, microbiology, and routine treatments including dornase alfa therapy. Recognition of these factors will improve the understanding of the ERCF database in preparation for future, more detailed analyses.

\section{Materials and methods}

\section{Inclusion of patients in the European Epidemiologic} Registry of Cystic Fibrosis

The methodology of the ERCF has been described previously [8, 9]. Briefly, nine countries (Austria, Belgium, Denmark, France, Germany, Ireland, the Netherlands, Sweden and the UK) provided demographic, diagnostic, genotypic and clinical data on patients diagnosed with CF. From its founding in 1994-1998, data pertaining to individual patient visits were collected on paper forms completed by participating clinics, which agreed to enrol into ERCF all the $\mathrm{CF}$ patients in their care. In total, over half of the known CF patients in Europe were enrolled.

The data collection forms were sent by the clinics to the clinical research organization, Quintiles $\mathrm{GmbH}$ in Neu Isenburg, Germany, for data entry, quality control and assignment of preferred terms. Over 400 semi-automated context and longitudinal consistency checks were performed on entered data and queries were issued to the clinical site, if necessary, to resolve inconsistencies. Only data that passed these checks were loaded into the ERCF database.

Beginning in 1997, a remote data entry (RDE) system, to which centres were gradually transferred following training and a thorough review of past data, was introduced using Internet technology. The RDE incorporated the context and consistency checks during data entry at the clinic, and performed most of the coding automatically. Final semi-automated consistency checks to ensure data quality were performed by Quintiles before the data were loaded into the ERCF database.

The following information was recorded at enrolment: demographics, features of the CF diagnosis, all routine therapies and current antibiotic regimens, physical status, medical history, microbiology and, if available, the most recent spirometry measurements.

\section{Definition of the analysis population}

Patients were included in the analyses if their enrolment data were included in the ERCF database by January 1998, and if they had a valid value of FEV1 recorded at enrolment or within the following 180 days and were aged $\geqslant 6$ yrs at enrolment. The first value of FEV1, within the 180-day interval was chosen if it yielded FEV1 \% pred using the Knudson method [10]. Results were examined for age-specific associations. Analysis parameters included clinical conditions or complications, signs and symptoms, micro-organisms isolated and treatments, which were considered present if reported at least once during the 180-day interval.
The specific factors that were assessed for their association with FEV1 \% pred values were: demographic data (sex, age, weight, height); clinical conditions and complications (asthma-like symptoms, allergic bronchopulmonary aspergillosis (ABPA), congestive heart failure, diabetes, gastro-oesophageal reflex, haemoptysis, malnutrition, nasal polyposis, pneumothorax); clinical signs and symptoms (clubbing, daily cough/sputum, crepitations, hyperinflation, wheezing); microbiology (Burkholderia cepacia, Pseudomonas aeruginosa, Haemophilus influenzae, Staphylococcus aureus, Aspergillus spp., Candida spp.); routine therapies (antibiotics, bronchodilators, corticosteroids, dornase alfa (Pulmozyme $\mathbb{R}$, Roche, Basel, Switzerland), nonsteroidal anti-inflammatory drugs (NSAIDs), physiotherapy and regular exercise).

\section{Reporting conventions}

The concept of statistical significance is not valid for population data because the sampling prerequisites for testing a null hypothesis are not met. Rather, the $95 \%$ confidence limits of the estimate of a mean value become narrower the larger the subgroup of the population studied and can result in comparisons between large subgroups, in apparent statistical significance with no clinical relevance. Furthermore, the large number of comparisons in the descriptive data implies that $5 \%$ of these will be found to be only spuriously statistically significant. The results reported here concern comparisons where the $95 \%$ confidence limits of the mean FEV1 values compared did not overlap. Thus, if statistical testing were to be (inappropriately) applied, all differences in FEV1 would achieve statistical significance. However, differences of $<5 \%$ pred between group means are reported as "not different" because of dubious clinical relevance.

Data were analysed for three age groups: 6-12 yrs, $13-17$ yrs and $\geqslant 18$ yrs. Differences of $>10 \%$ and $5-10 \%$ in the FEV1 \% pred between patients with and without individual factors were reported because of their potential clinical importance. Subgroup analyses were performed on patient populations numbering 210; patient groups of $<10$ were not included in the FEV1 analyses. Oral quinolones and all antibiotics administered parenterally or by inhalation were classified as antipseudomonal antibiotics; oral antibiotics excluding quinolones were classified as oral antibiotics. Weight for age was expressed as a percentile of ideal weight for age [11] and was analysed according to quartiles of the population. Malnutrition was also examined according to the use of enteral or parenteral nutrition.

\section{Results}

\section{Patient population}

Of the 12,084 patients enrolled in the ERCF by January 1998, 7,010 patients aged $\geqslant 6$ yrs (females, $\mathrm{n}=3,299(47 \%)$; males, $\mathrm{n}=3,711(53 \%))$, who had a 
Table 1. - Forced expiratory volume in one second (FEV1) $\%$ pred by weight for age percentiles for overall European Epidemiologic Registry of Cystic Fibrosis patient population aged $>6$ yrs

\begin{tabular}{lccc}
\hline \multirow{2}{*}{$\begin{array}{l}\text { Weight for } \\
\text { age percentile }\end{array}$} & $\begin{array}{c}\text { Patients contributing } \\
\text { valid FEV1 values } \mathrm{n}\end{array}$ & \multicolumn{2}{c}{ FEV1 \% pred } \\
\cline { 3 - 4 } & & Mean & $95 \%$ CI \\
\hline$<6$ & 1767 & 52.0 & $50.7-53.3$ \\
$6-<23$ & 1839 & 64.0 & $62.8-65.1$ \\
$23-<50$ & 1677 & 73.2 & $72.1-74.3$ \\
$\geqslant 50$ & 1721 & 78.2 & $77.1-79.3$ \\
\hline
\end{tabular}

CI: confidence interval.

valid FEV1 value at enrolment or within 180 days of enrolment were analysed. Excluded were 4,711 patients with no FEV1 \% pred value calculable within the baseline time window, and an additional 363 patients aged $<6$ yrs who reported baseline FEV1. Patients were subdivided into three age groups: $6-12$ yrs $(n=2,602), 13-17$ yrs $(n=1,655)$ and $\geqslant 18$ yrs $(n=2,753)$.

\section{Age group}

Cross-sectional analysis of the three age groups demonstrated a progressively lower FEV1 \% pred with advancing age. Whereas children 6-12 yrs of age showed a near normal mean value of FEV1\% pred $(79.1 \%)$, patients in the $13-17$ yrs and $\geqslant 18$ yrs age groups showed mean values of $67.8 \%$ and $54.1 \%$, respectively.

Sex

In contrast to age, sex did not demonstrate an obvious association with $\mathrm{FEV}_{1} \%$ pred. The mean values for males and females were $80.1 \%$ and $78.1 \%$ in the $6-12$ yrs age group, $67.8 \%$ and $67.8 \%$ in the
$13-18$ yrs age group, and $53.5 \%$ and $54.8 \%$ in the $\geqslant 18$ yrs age group, respectively.

\section{Weight for age percentiles}

Quartiles of the population fell approximately into the classes $<6$ th, 6 th $-\leqslant 23 \mathrm{rd}, 23 \mathrm{rd}-<50$ th and $\geqslant 50$ th percentiles. Analysis of the overall ERCF population demonstrated a strong association between poor respiratory function and weight for age percentiles in all age groups (table 1 ).

\section{Clinical conditions and complications}

Certain clinical conditions and complications were found to be associated with differences in the FEV1\% pred values at enrolment. Haemoptysis and use of enteral or parenteral nutrition (indicators of malnutrition) were associated with $>10 \%$ lower FEV1 in all age groups. Congestive heart failure, pneumothorax and diabetes mellitus increased in prevalence with age (table 2). The two former conditions were associated with a $>10 \%$ lower FEV1 in adolescent and/or adult patients, while diabetes mellitus was associated with a $5-10 \%$ lower FEV1 in those aged $>18$ yrs.

The prevalence of ABPA, asthma-like symptoms and gastro-oesophageal reflux was independent of age. These conditions were associated with $\sim 5-10 \%$ lower FEV1 values in all age groups. Asthma-like symptoms and haemoptysis were the most frequently reported conditions $(22.7 \%$ and $13.1 \%$, respectively) that were associated with lower FEV1. Although gall bladder disease and portal hypertension were associated with a $5-10 \%$ lower FEV1 in the overall population, subanalysis by age group suggested that this was primarily due to the age of the effected patients.

The only complication associated with a higher FEV1 \% pred value was nasal polyposis, reported in $14.3 \%$ of the patients (table 2). The difference was

Table 2. - Prevalence of clinical conditions and complications associated with differences in forced expiratory volume in one second $\left(F E V_{1}\right) \%$ pred

\begin{tabular}{|c|c|c|c|c|}
\hline \multirow[t]{2}{*}{ Clinical conditions and complications } & \multicolumn{3}{|c|}{ Age } & \multirow[t]{2}{*}{ Overall } \\
\hline & $6-12$ yrs & $13-17 \mathrm{yrs}$ & $\geqslant 18$ yrs & \\
\hline Subjects n & 2602 & 1655 & 2753 & 7010 \\
\hline \multicolumn{5}{|l|}{$>10 \%$ lower FEV $1 \%$ pred } \\
\hline Congestive heart failure & $6(0.2)^{*}$ & $7(0.4)^{*}$ & $23(0.8)$ & $23(0.3)$ \\
\hline Haemoptysis & $99(3.8)$ & $187(11.3)$ & $631(22.9)$ & $917(13.1)$ \\
\hline Indicators of malnutrition & $149(5.7)$ & $140(8.5)$ & $164(6.0)$ & $453(6.5)$ \\
\hline Pneumothorax & $7(0.3)^{*}$ & $23(1.4)$ & $83(3.0)$ & $106(1.5)$ \\
\hline \multicolumn{5}{|l|}{$5-10 \%$ lower FEV1 \% pred } \\
\hline ABPA & $173(6.7)$ & $131(7.9)$ & $215(7.8)$ & $519(7.4)$ \\
\hline Asthma-like symptoms & $545(20.9)$ & $373(22.5)$ & $672(24.4)$ & $1590(22.7)$ \\
\hline Diabetes melliuts & $12(0.5)$ & $42(2.5)$ & $161(5.8)$ & $215(3.1)$ \\
\hline Gastro-oesophageal reflux & $96(3.7)$ & $77(4.7)$ & $181(6.6)$ & $354(5.0)$ \\
\hline \multicolumn{5}{|l|}{$5-10 \%$ higher FEV $1 \%$ pred } \\
\hline Nasal polyposis & $366(14.1)$ & $264(15.9)$ & 375 (13.6) & $1005(14.3)$ \\
\hline
\end{tabular}

Data are presented as n (\%). ABPA: allergic bronchopulmonary aspergillosis. *: Patient prevalence $<10$ is not included in the analysis of FEV1. 
Table 3. - Prevalence of clinical signs and symptoms at time of enrolment associated with differences in forced expiratory volume in one second $\left(\mathrm{FEV}_{1}\right) \%$ pred

\begin{tabular}{lccrr}
\hline Signs and symptoms & \multicolumn{3}{c}{ Age } & Overall \\
\cline { 2 - 3 } & $6-12$ yrs & $13-17$ yrs & $\geqslant 18$ yrs \\
\hline Subjects n & 2602 & 1655 & 2753 & 7010 \\
$>10 \%$ lower FEV1 \% pred & & & & \\
Daily cough & $1416(54.4)$ & $1164(70.3)$ & $2374(86.2)$ & $4954(70.7)$ \\
Daily sputum & $1184(45.5)$ & $1084(65.5)$ & $2323(84.4)$ & $4591(65.5)$ \\
Presence of clubbing & $1075(41.3)$ & $866(52.3)$ & $1650(59.9)$ & $3591(51.2)$ \\
Crepitations & $728(28.0)$ & $705(42.6)$ & $1547(56.2)$ & $2980(42.5)$ \\
Hyperinflation & $799(30.7)$ & $575(34.7)$ & $843(30.6)$ & $2217(31.6)$ \\
Wheezing & $386(14.8)$ & $278(16.8)$ & $683(24.8)$ & $1347(19.2)$ \\
\hline
\end{tabular}

Data are presented as n (\%).

modest $(5-10 \%)$ and was seen only in patients $>12 \mathrm{yrs}$ of age. There were no differences in mean FEV1 \% pred values for other clinical conditions such as mild liver disease, distal intestinal obstructive syndrome and cholelithiasis.

\section{Clinical signs and symptoms}

The prevalence of various clinical signs and symptoms associated with lower FEV1 \% pred values at enrolment are shown in table 3. The presence of pulmonary symptoms such as daily cough or sputum, crepitations, clubbing, hyperinflation or wheezing was in each, associated with a $>10 \%$ lower FEV 1 in patients of all age groups than in patients not reporting these symptoms. The most and least prevalent signs were daily cough and wheezing, with an overall occurrence of $70.7 \%$ and $19.2 \%$, respectively.

\section{Microbiology}

Of the 7,010 patients $\geqslant 6$ yrs of age with baseline FEV1 determinations, 6,774 (96.6\%) had microbiological data available for the 180-day sampling period. Some pathogens isolated from sputum, throat or bronchoalveolar lavage fluid were associated with differences in FEV1 \% pred values. $P$. aeruginosa was associated with a $>10 \%$ lower FEV1 across the three age groups compared with patients without this infection. The overall prevalence of $P$. aeruginosa, the most commonly isolated pathogen in ERCF patients, was 4,592/6,774 (67.8\%); and the proportion of patients with positive cultures increased from $54.2 \%$, in the $6-12$ years age group, to $78.8 \%$ in the $\geqslant 18$ yrs age group (table 3 ). Similarly, B. cepacia was associated with $\sim 10 \%$ lower FEV1 in all age groups. However, it was isolated from only $5.6 \%$ of patients, mostly in the older age groups (table 4).

Both Aspergillus and Candida colonization were associated with $\sim 5-10 \%$ lower FEV1 in all age groups and were isolated from $16.9 \%$ and $33.5 \%$ of patients, respectively (table 4). In contrast, infection with either $S$. aureus or $H$. influenzae was not associated with low FEV1 \% pred. An apparent association in the overall population of $H$. influenzae with a higher FEV1 \% pred disappeared when patients were stratified by age. Since the per cent of patients reporting $H$. influenzae decreased with increasing age (table 4 ), the difference seen in the overall population probably reflects the younger age of patients reporting $H$. influenzae.

Findings regarding antibiotic usage were consistent with those concerning the organisms for which they are indicated. Antipseudomonal antibiotics (quinolones and antibiotics given by inhaled or intravenous

Table 4. - Prevalence of colonizing micro-organisms associated with differences in forced expiratory volume in one second (FEV1) \% pred

\begin{tabular}{|c|c|c|c|c|}
\hline \multirow[t]{2}{*}{ Colonizing micro-organism } & \multicolumn{3}{|c|}{ Age } & \multirow[t]{2}{*}{ Overall } \\
\hline & $6-12$ yrs & $13-17$ yrs & $\geqslant 18 \mathrm{yrs}$ & \\
\hline $\begin{array}{l}\text { Subjects n } \\
>10 \% \text { lower FEV } 1 \% \text { pred }\end{array}$ & 2511 & 1590 & 2673 & 6774 \\
\hline $\begin{array}{l}\text { Burkholderia cepacia } \\
\text { Pseudomonas aeruginosa }\end{array}$ & $\begin{aligned} & 66(2.6) \\
& 1360(54.2)\end{aligned}$ & $\begin{array}{c}89(5.6) \\
1126(70.8)\end{array}$ & $\begin{array}{c}224(8.4) \\
2106(78.8)\end{array}$ & $\begin{array}{c}379(5.6) \\
4592(67.8)\end{array}$ \\
\hline $5-10 \%$ lower FEV1 \% pred & & & & \\
\hline $\begin{array}{l}\text { Aspergillus spp. } \\
\text { Candida } \text { spp. }\end{array}$ & $\begin{array}{l}362(14.4) \\
756(30.1)\end{array}$ & $\begin{array}{l}297(18.7) \\
536(33.7)\end{array}$ & $\begin{array}{l}485(18.1) \\
935(35.0)\end{array}$ & $\begin{array}{l}1144(16.9) \\
2227(33.5)\end{array}$ \\
\hline No difference in FEV $1 \% \mathrm{pr}$ & & & & \\
\hline Haemophilus influenzae & 995 (39.6) & $420(26.4)$ & 496 (18.6) & $1911(28.2)$ \\
\hline Staphylococcus aureus & $1479(58.9)$ & $954(60.0)$ & $1389(52.0)$ & $3822(56.4)$ \\
\hline
\end{tabular}

Data are presented as $\mathrm{n}(\%)$. 
routes) were associated with $>10 \%$ lower FEV1 values, while oral antibiotics (except quinolones) commonly used against Staphylococcus and Haemophilus, were not.

Routine treatments and their association with forced expiratory volume in one second

Several intensive routine therapies, including oral corticosteroids, NSAIDs, dornase alfa, oxygen and assisted ventilation, were used in patients of all age groups who had a mean FEV1 \% pred $>10 \%$ lower than untreated patients (table 5). Oral bronchodilators and vitamins were associated with only a $5-10 \%$ difference in pulmonary function. Use of airway clearance, inhaled bronchodilators, oral nutritional supplements and pancreatic enzymes were associated with $>10 \%$ lower FEV1 only in patients $>12$ yrs of age, and with 5-10\% lower FEV1 values in the youngest age group. Inhaled corticosteroids and insulin or oral hypoglycaemics were both associated with $5-10 \%$ lower FEV 1 only in patients $>12$ yrs old, while the latter were prescribed primarily for these patients (table 5). Diuretic use was also age-dependent and associated with $>10 \%$ lower FEV1 only in patients aged $>12$ yrs. The other treatments were used frequently across all age groups (table 5). No association was found between FEV1 \% pred values and the use of mucolytics/expectorants or ursodeoxycholate.

Although regular exercise was associated with $5-10 \%$ better FEV1 in the overall population, analyses by age groups demonstrated that this association occurred only in adults.

\section{Discussion}

Age and sex

As expected, age had a major influence on FEV1\% pred, with progressively poorer lung function associated with increasing age. Therefore, associations were sought in patients stratified into age groups of $6-<13$ yrs (children), 13-<18 yrs (adolescents) and $\geqslant 18$ yrs (adults).

There was no obvious difference in pulmonary status between males and females at any age, although fewer females than males were included in the analyses. While this is consistent with other studies [5], it does not support the hypothesis that females are at greater risk of poorer weight maintenance, as suggested by a large mortality study [6, 12, 13], because poorer nutrition is strongly associated with lower FEV1 in ERCF. Therefore, any impact of sex on mortality is likely to be independent of pulmonary function.

Association of forced expiratory volume in one second $\%$ predicted with malnutrition

Review of the weight for age percentiles revealed a strong association with FEV1: mean FEV1 values were $52.0 \%$ and $64.0 \%$ for the $<6$ th and 6 th $-<23 \mathrm{rd}$ weight for age percentiles, respectively (table 1). Analyses of the weight/height data in the ERCF were consistent with those of others [14], based on the National Cystic Fibrosis Registry data, which established that $20 \%$ of patients were below the 5 th percentile for height and weight for age. The percentage

Table 5. - Prevalence of routine treatments associated with differences in forced expiratory volume in one second (FEV 1$)$ $\%$ pred

\begin{tabular}{|c|c|c|c|c|}
\hline \multirow[t]{2}{*}{ Routine treatment } & \multicolumn{3}{|c|}{ Age } & \multirow[t]{2}{*}{ Overall } \\
\hline & $6-12$ yrs & $13-17$ yrs & $\geqslant 18$ yrs & \\
\hline $\begin{array}{l}\text { Subjects } \mathrm{n} \\
>10 \% \text { ower FEV1 } \% \text { pred for all age orouns }\end{array}$ & 2602 & 1655 & 2753 & 7010 \\
\hline $\begin{array}{l}>10 \% \text { lower FEV } 1 \% \text { pred for all age groups } \\
\text { Antipseudomonal antibiotics* }\end{array}$ & $936(36.0)$ & $856(51.7)$ & $1530(55.6)$ & $3322(47.4)$ \\
\hline Dornase alfa & $917(35.2)$ & $753(45.5)$ & $1277(46.4)$ & $2947(42.0)$ \\
\hline Oral corticosteroids & $205(7.9)$ & 197 (11.9) & $482(17.5)$ & $884(12.6)$ \\
\hline Oxygen & $65(2.5)$ & $83(5.0)$ & $273(9.9)$ & $421(6.0)$ \\
\hline NSAIDs & $33(1.3)$ & $36(2.2)$ & $96(3.5)$ & $165(2.3)$ \\
\hline Nasal ventilation (assisted) & $17(0.7)$ & $23(1.4)$ & $32(1.2)$ & $72(1.0)$ \\
\hline \multicolumn{5}{|l|}{$>10 \%$ lower FEV $1 \%$ pred for age groups $>12$ yrs } \\
\hline Airway clearance & $2463(94.7)$ & 1519 (91.2) & $2368(86.0)$ & $6350(90.6)$ \\
\hline Diuretics & $7(0.3)^{+}$ & $15(0.9)$ & $52(1.9)$ & $74(1.1)$ \\
\hline Inhaled bronchodilators ${ }^{\#}$ & $1485(57.1)$ & $1104(66.7)$ & $2044(74.2)$ & $4633(66.1)$ \\
\hline Oral nutritional supplements & $1178(45.3)$ & $726(43.9)$ & $1014(36.8)$ & 2918 (41.6) \\
\hline Pancreatic enzymes & $2500(96.1)$ & $1579(95.4)$ & $2487(90.3)$ & $6566(93.7)$ \\
\hline \multicolumn{5}{|l|}{$5-10 \%$ lower FEV $1 \%$ pred for age groups $>12$ yrs } \\
\hline Inhaled corticosteroids & $720(27.7)$ & $599(36.2)$ & 1099 (39.9) & $2418(34.5)$ \\
\hline Insulin or oral hypoglycaemics & $40(1.5)$ & $132(8.0)$ & $475(17.3)$ & $647(9.2)$ \\
\hline \multicolumn{5}{|l|}{$5-10 \%$ higher FEV $1 \%$ pred for age group $\geqslant 18$ yrs } \\
\hline Regular exercise & $1316(50.6)$ & $848(51.2)$ & $1346(48.9)$ & $3510(50.1)$ \\
\hline
\end{tabular}

Data are presented as n (\%). NSAIDs: nonsteroid anti-inflammatory drugs. *: oral quinolones and all nonoral antibiotics; ${ }^{+}$: patient prevalence $<10$ is not included in the analysis; " : Oral bronchodilators were associated with only a 5-10\% lower FEV1 $\%$ pred at all ages. 
of the ideal weight was a highly significant individual factor for predicting mortality in the Canadian Patient Data Registry [6], but when FEV1 and sex were introduced into the analysis model, no correlation of weight with mortality was observed. This suggests a relationship between low weight for age and poor pulmonary function.

When malnutrition was alternatively identified by the use of enteral or more rarely parenteral nutritional support, it remained a major factor associated with lower FEV1 \% pred values. Malnutrition has already been shown to be a major predictive factor for mortality $[5,6]$. The benefit of a high-energy, highfat diet, together with the administration of a new generation of enteric-coated pancreatic enzymes, was clearly demonstrated $>10$ yrs ago [12]. Since then, several studies have reported a close relationship between nutritional status and the degree of respiratory impairment, as well as the ability of nutritional supplements to improve pulmonary function in patients with mild-to-moderate pulmonary impairment [15-17]. Although the present analysis confirms these observations, it is unable to clarify the causality of the relationship between nutrition and pulmonary function.

Association between clinical conditions and complications and forced expiratory volume in one second $\%$ predicted

Pneumothorax, haemoptysis and congestive heart failure were important factors associated with lower FEV1 values within each age group, as might be expected from the increasing frequency of these complications in advanced lung disease. Interestingly, slightly lower FEV1 values were observed with gastrooesophageal reflux, suggesting that the importance of this condition may be underestimated. However, the ERCF provided no definition of gastrooesophageal reflux and consequently this potential relationship remains to be confirmed.

Diabetes mellitus was associated with lower FEV1 in patients $\geqslant 18 \mathrm{yrs}$ old, suggesting that diabetes mellitus is another metabolic complication associated with a poor prognosis for adult CF patients. The significant increase in mortality reported in $\mathrm{CF}$ patients with diabetes mellitus [18] may be the most compelling evidence for this association. The decline in pulmonary function in patients with diabetes mellitus has been associated with colonization of the respiratory tract by $P$. aeruginosa [18], although it is more frequently correlated with weight loss and restricted inspiratory muscle capacity than with a worsening $P$. aeruginosa infection [19]. ERCF data support the impact of diabetes mellitus on pulmonary function but not an important intermediary role for $P$. aeruginosa (unpublished data).

Unexpectedly, asthma-like symptoms and ABPA were associated with only $5-10 \%$ lower FEV1. This may reflect the reversibility of these medical conditions with time and the efficacy of treatments that allow recovery of pulmonary function after acute illness. The results relating to bronchial hyperactivity can be compared with those of other studies [20, 21], which were unable to demonstrate a relationship between the presence of bronchial hyperactivity and the severity of CF. The ABPA results from the ERCF data have been analysed in detail by MASTELLA et al. [9].

As expected, gall bladder disease, which is common in CF patients, was not associated with poor pulmonary function when results were corrected for age. Similarly, portal hypertension and elevated liver function tests were not associated with lower FEV1 \% pred values, despite reports from previous studies indicating that these conditions may affect pulmonary circulation or mortality [22, 23]. This is consistent with a recent prospective study of liver disease over 15 yrs [24], in which an influence on lung function could not be confirmed.

The observation that nasal polyposis was associated with better pulmonary status has been reported previously [25]. However, no scientific rationale has been proposed to date for the modest negative association between nasal polyps and the severity of lung disease in adolescents and adults. This observation suggests that the presence or absence of nasal polyps may be a prognostic indicator in CF patients $[8,25]$.

Association between clinical signs and symptoms and forced expiratory volume in one second $\%$ predicted

At the time of enrolment, the presence of pulmonary symptoms such as daily cough, daily sputum, wheezing, crepitations, clubbing and hyperinflation were each associated with $>10 \%$ lower FEV 1 across all age groups. Clinically, this suggests that the medical team should always consider pulmonary symptoms when choosing treatment for CF patients. Appropriate symptomatic therapy is likely to improve treatment outcome and, in turn, have a positive effect on patient compliance.

\section{Microbiological data associated with lower forced expiratory volume in one second \% predicted}

The ERCF data indicated that sputum cultures positive for $P$. aeruginosa or $B$. cepacia were each associated with $>10 \%$ lower FEV1 values in all age groups. These pathogens have been identified as highly significant negative predictive factors in a previous study based on data from the Canadian Patient Data Registry [6]. Pulmonary colonization by $P$. aeruginosa appears to be closely related to pulmonary decline, and mean FEV $1 \%$ pred has previously been reported to be significantly lower in patients with pulmonary $P$. aeruginosa infection (49\%) than in patients without infection $(82.8 \%$, ) [26]. Although B. cepacia infection was also associated with a lower FEV1 \% pred, the degree of colonization has been reported to be unrelated [26].

Sputum cultures positive for Aspergillus spp. and Candida spp. were associated with 5-10\% lower FEV1 at all ages. Although sputum cultures positive for 
$H$. influenzae appeared to be associated with higher FEV1 \% pred values overall, this difference disappeared when patients were stratified by age. $S$. aureus infection was associated with no difference in the mean FEV1 \% pred value. While $H$. influenzae infection is commonly observed in the sputum of young patients at the initial phase of the disease and is considered less aggressive [27-29], the opposite is thought to be true for $S$. aureus. The reputation of Staphylococcus spp. as an active, aggressive pathogen is debatable, but it is well established that some patients with chronic $S$. aureus infection exhibit a decline in pulmonary function comparable to that observed with $P$. aeruginosa colonization; this may even result in the need for lung transplantation. Although Hoiby [30] reported that the presence of $S$. aureus infection in CF patients $<13$ yrs of age was correlated with a lower FEV1 \% pred, the use of prophylactic antistaphylococcal therapy in young patients remains controversial [31, 32]. While the present, simple cross-sectional analysis does not support a major impact of $S$. aureus on pulmonary function, it by no means rules out the possibility that this organism may contribute to pulmonary dysfunction in a manner not yet fully understood.

Treatment of cystic fibrosis patients based on pulmonary status

Confirming an earlier report from ERCF [8], most aggressive routine therapies were found to be prescribed preferentially to patients with lower FEV1 \% pred in all age groups. This is expected for assisted nasal ventilation and oxygen therapies that are given routinely to $\mathrm{CF}$ patients with a poorer pulmonary status, but it was also observed with certain antiinflammatory drugs (e.g. oral corticosteroids and NSAIDs), dornase alfa and antipseudomonal antibiotics. In view of mounting evidence that inflammation is present early in $\mathrm{CF}$ lung disease, properly powered clinical trials are urgently needed to determine the place of these therapies in patients with relatively well-preserved lung function. One such trial recently reported clinical benefit from dornase alfa in children aged 6-10 yrs with forced vital capacity $>85 \%$ pred [33].

CF patients $>12$ yrs of age who were treated with pancreatic enzymes, airway clearance, inhaled bronchodilators and oral nutritional supplements also had lower FEV1; those receiving insulin or oral hypoglycaemics had slightly lower FEV1 values. Although one must be cautious when analysing cross-sectional data across different age groups, these data are consistent with the following interpretations. Since most CF patients $<18$-yrs-old receive pancreatic enzymes and adults $(\geqslant 18 \mathrm{yrs})$ receive this therapy only if required, those CF patients who never actually required pancreatic enzymes may have better outcomes in adulthood. The age-dependent association of insulin or oral hypoglycaemics with $5-10 \%$ lower FEV1 \% pred is probably due the age-dependent increase in the prevalence of diabetes (table 2), which itself demonstrated an association with poor pulmonary function.
Only adults performing regular exercise demonstrated higher FEV1. While this may support the efficacy of such treatment, it is more likely to reflect the ability of older patients to maintain an exercise regimen if they have better respiratory function. This interpretation is supported by a previous study [34] that demonstrated the capability of a training programme to increase work capacity and respiratory muscle endurance, although this was not correlated with a clear improvement in pulmonary function.

This European Epidemiologic Registry of Cystic Fibrosis study has analysed a large number of patients and has validated previous findings from smaller samples that may not have been truly representative of the populations from which they came. Although cross-sectional analysis cannot establish cause and effect, it is significant that this extensive European database supports much previous work and generates new hypotheses that should be included in statistical modelling of observational data or tested in multicentre randomized clinical trials. To the authors' knowledge, this is the first epidemiological analysis of this size and nature to have been performed for cystic fibrosis in Europe.

\section{References}

1. FitzSimmons SC. The changing epidemiology of cystic fibrosis. J Pediatr 1993; 122: 1-9.

2. Collins FS. Cystic fibrosis: molecular biology and therapeutic implications. Science 1992; 256: 774-779.

3. Riordan JR. The cystic fibrosis transmembrane conductance regulator. Апnи Rev Physiol 1993; 55: 609-630.

4. FitzSimmons SC. Cystic Fibrosis Foundation Patient Registry 1997 Annual Data Report. Bethesda, Maryland, 1998.

5. Huang NN, Schidlow DV, Szatrowski TH, et al. Clinical features, survival rate, and prognostic factors in young adults with cystic fibrosis. Am J Med 1987; 82: 871-879.

6. Corey M, Farewell V. Determinants of mortality in cystic fibrosis in Canada 1970-89. Am J Epidemiol 1996; 143: 1007-1017.

7. Kerem E, Reisman J, Corey M, Canny GJ, Levison H. Prediction of mortality in patients with cystic fibrosis. $N$ Engl J Med 1992; 326: 1187-1191.

8. Koch C, McKenzie SG, Kaplowitz H, et al. International practice patterns by age and severity of lung disease in cystic fibrosis: data from the Epidemiologic Registry of Cystic Fibrosis (ERCF). Pediatr Pulmonol 1997; 24: 147-154.

9. Mastella G, Rainisio M, Harms HK, et al. Allergic bronchopulmonary aspergillosis in cystic fibrosis. A European epidemiological study. Eur Respir J 2000; 16: $464-471$.

10. Knudson RL, Lebowitz MD, Holberg CJ, Burrows B. Changes in the normal maximal expiratory flowvolume curve with growing and aging. Am Rev Respir Dis 1983; 127: 725-734.

11. Hamill PVV, Drizd TA, Johnson CL, et al. Physical growth: National Center for Health Statistics percentiles. Am J Clin Nutr 1979; 32: 607-629.

12. Corey M, McLaughlin FJ, Williams M, Levison H. A 
comparison of survival, growth and pulmonary function in patients with cystic fibrosis in Boston and Toronto. J Clin Epidemiol 1988; 41: 583-591.

13. Rosenstein BJ, Zeitlin PL. Prognosis in cystic fibrosis. Curr Opin Pulm Med 1995; 1: 444449.

14. Lai HC, Kosorok MR, Sondel SA, et al. Growth status in children with cystic fibrosis based on the National Cystic Fibrosis Patient Registry data: evaluation of various criteria used to identify malnutrition. J Pediatr 1998; 132: 478-485.

15. Shepherd R, Cooksley WG, Cooke WD. Improved growth and clinical, nutritional, and respiratory changes in response to nutritional therapy in cystic fibrosis. J Pediatr 1980; 97: 351-357.

16. Moore MC, Greene HL, Donald WD, Dunn GD. Enteral-tube feeding as adjunct therapy in malnourished patients with cystic fibrosis: a clinical study and literature review. Am J Clin Nutr 1986; 44: 33-41.

17. Gaskin KJ. The impact of nutrition in cystic fibrosis: a review. J Pediatr Gastroenterol Nutr 1988; 7: Suppl. 1, S12-S17.

18. Finkelstein SM, Wielinski CL, Elliott GR, et al. Diabetes mellitus associated with cystic fibrosis. J Pediatr 1988; 112: 373-377.

19. Lanng S, Thorsteinsson B, Nerup J, Koch C. Influence of the development of diabetes on clinical status in patients with cystic fibrosis. Eur J Pediatr 1992; 151: 684-687.

20. Valleta E, Braggioni C, Mastella G. Desensitization to Aspergillus and allergic bronchopulmonary aspergillosis in a cystic fibrosis population. Pediatr Asthma Allergy Immunol 1993; 7: 43-49.

21. Light M, Kaplowitz H, Geller D, Colin A. Prevalence of allergic bronchopulmonary aspergillosis at enrollment into the epidemiologic study of cystic fibrosis. Pediatr Pulmonol 1995; 12: Suppl. 12, 28.

22. Whitington PF, Balistreri F. Liver transplantation in paediatrics: indications, contraindications and pretransplant management. J Pediatr 1991; 118: 169-177.

23. Williams SG, Hayller KM, Hodson ME, Westaby D. Prognosis in cystic fibrosis. N Engl J Med 1992; 327: 1244-1245.
24. Lindblad A, Glaumann H, Strandvik B. Natural history of liver disease in cystic fibrosis. Hepatology 1999; 30: 1151-1158.

25. Kingdom TT, Lee KC, FitzSimmons SC, Cropp GJ. Clinical characteristics and genotype analysis of patients with cystic fibrosis and nasal polyposis requiring surgery. Arch Otolaryngol Head Neck Surg 1996; 122: 1209-1213.

26. Ballestero S, Escobar H, Villaverde R, et al. Microbiological parameters and clinical evolution in cystic fibrosis. In: Escobar H, Baquero F, Suarez L, eds. Clinical Ecology of Cystic Fibrosis. Elsevier Science, 1993; pp. 55-62.

27. Hoiby N, Kilian M. Haemophilus from the lower respiratory tract of patients with cystic fibrosis. Scand J Respir Dis 1976; 57: 103-107.

28. Hoiby N. Microbiology of lung infections in cystic fibrosis patients. Acta Paediatr Scand 1982; 301: 3354.

29. Le Bourgeois M, Gerardin M, Munck A, Navarro J. Mucoviscidosis. Antibiotic management. Presse Med 1997; 26: 727-732.

30. Hoiby N. Prevention and treatment of infections in cystic fibrosis. Int J Antimicrobiol Agents 1992; 1: 229238.

31. Weaver LT, Green MR, Nicholson K, et al. Prognosis in cystic fibrosis treated with continuous flucloxacillin from the neonatal period. Arch Dis Child 1994; 70: 84 89.

32. Stutman H. To Keflex or not to Keflex: long-term antibiotics in CF. 9th Annual North American CF Conference, Dallas, Texas, 1995.

33. Konstan MW, Tiddens HA, Quan JM, et al. A randomized, placebo-controlled trial of two years'

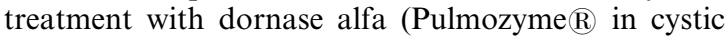
fibrosis patients aged 6-10 years with early lung disease. Pediatr Pulmonol 2000; Suppl. 20, S299-S300.

34. Orenstein DM, Franklin BA, Doershuk CF, et al. Exercise conditioning and cardiopulmonary fitness in cystic fibrosis. The effects of a three-month supervised running program. Chest 1981; 80: 392-398. 